\title{
Dual-Band Notch Filter Based on Twist Split Ring Resonators
}

\author{
Haibin Sun, ${ }^{1}$ Cai Feng, ${ }^{2}$ Yongjun Huang, ${ }^{1}$ Ruiming Wen, ${ }^{1}$ Jian Li, ${ }^{1}$ \\ Weijian Chen, ${ }^{1}$ and Guangjun Wen ${ }^{1}$ \\ ${ }^{1}$ Key Laboratory of Broadband Optical Fiber Transmission \& Communication Networks, \\ Centre for RFIC and System Technology, School of Communication and Information Engineering, \\ University of Electronic Science and Technology of China, Chengdu 611731, China \\ ${ }^{2}$ School of Electronic and Information Engineering, Beijing University of Aeronautics and Astronautics, Beijing 100191, China
}

Correspondence should be addressed to Yongjun Huang; yongjunh@uestc.edu.cn

Received 9 December 2013; Revised 8 February 2014; Accepted 18 February 2014; Published 30 April 2014

Academic Editor: Francisco Falcone

Copyright (C) 2014 Haibin Sun et al. This is an open access article distributed under the Creative Commons Attribution License, which permits unrestricted use, distribution, and reproduction in any medium, provided the original work is properly cited.

A novel dual-band rectangular waveguide notch filter is experimentally investigated in this paper. Such filter is realized by integrating two pairs of split ring resonators (SRRs) printed on the two sides of a dielectric slab with twist angles and separated as a distance in the center of the rectangular waveguide. Due to the coupling effects between the twist SRRs and between the original SRRs and their mirror images generated by the metallic walls perpendicular to the E-field direction, it can flexibly contribute two disjunct resonance states and result in the dual-band notch properties. Furthermore, the two resonance frequencies can be controlled by changing the twist angles, resulting in the shifts of notch frequency bands.

\section{Introduction}

Metamaterial [1] is a kind of man-made structural composite, firstly predicted by Veselago in 1968 [2] and experimentally realized in 2000 by Smith et al. [3], by composing split ring resonator (SRR) [4] and continuous wire array [5]. Metamaterial exhibits some novel electromagnetic and optical properties which are not found in nature media, for example, negative refraction, reversals of both Doppler shift and Cherenkov radiation [2], enhancement of evanescent wave, and subwavelength imaging [6]. These novel properties have been experimentally demonstrated in recent years [711] and therefore various application proposals have been reported and analyzed [12-16].

In particular, recently, the microwave engineering applications of artificial metamaterials have been widely discussed and various novel microwave components, for example, filters [17], shifters [18], multiplexers [19], and power combiners/dividers [20], were reported, since the first realization of metamaterials [3]. The metamaterials based filters are mostly realized by integrating metamaterial resonators into conventional transmission systems so that the filters can pass or stop electromagnetic waves [21], and some metamaterialinspired filters operated at terahertz frequency bands have been reported $[22,23]$. Specifically, due to the single negative permeability or permittivity characteristics of some metamaterials such as SRRs and electric- $L C$ resonators, the electromagnetic waves or signals cannot be transmitted in such media and therefore the band stop (notch) filters [2426] are achieved.

On the other hand, it has been demonstrated that the rectangular waveguide loaded with SRR in its center position would lead to a transmission dip nearby the resonant frequency of SRR [27]. Quite recently, Zhang et al. analyzed that, due to the coupling effect between the original SRR and its mirror image generated by the metallic walls perpendicular to the E-field direction, the resonance frequency (also the notch frequency) can be shifted by changing the positions along the E-field direction [28]. In our previous works, we have systematically analyzed the coupling effects of SRRs and their mirror images in a rectangular waveguide through theoretical analysis and experimental measurements [26]. Such couplings can be manipulated mechanically by rotating the SRRs along a dielectric rod and/or shifting the SRRs up/down along the sidewall of the rectangular waveguide, resulting in shifts of the resonant frequencies and modulations of the resonant magnitudes. 


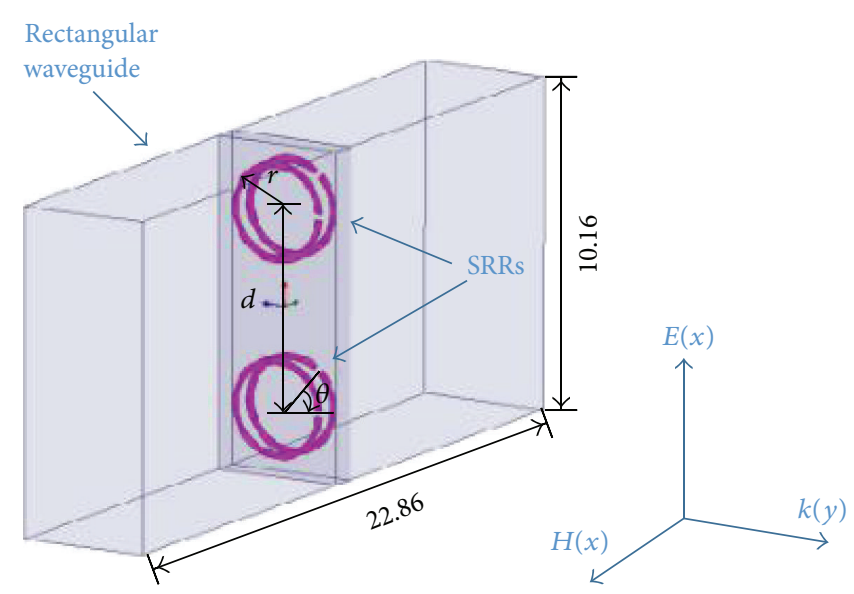

FIGURE 1: Schematic geometry of the proposed notch filter.

Among the developed band notch filters, however, they are mostly single band configurations. Actually, we found that when two pairs of SRRs printed on the two sides of a dielectric slab with twist angles and separated as a distance along the E-field direction in the rectangular waveguide (as shown in Figure 1), two resonance states would occur. This is because when two pairs of twist SRRs are placed in the rectangular waveguide with configuration as shown in Figure 1, the upper twist SRRs are coupled to the top edge of rectangular waveguide and simultaneously the nether one is coupled to the bottom edge. Moreover, the relative positions of the splits for the two pairs of SRRs compared to the top and bottom edges of rectangular waveguide have different values. It results in the different coupling values and therefore two resonance frequencies (notch frequencies) are obtained. In this paper, a schematic configuration is presented to show the dual-band notch filter realization and the controllability of the working frequencies is also analyzed.

\section{Design and Fabrications}

The schematic geometry of the proposed dual-band rectangular waveguide notch filter is shown in Figure 1. The metallic SRRs with width of $0.2 \mathrm{~mm}$ and thickness of $0.017 \mathrm{~mm}$ are printed on the two sides of a Rogers 4003 substrate (dielectric constant $\epsilon_{r}=3.55$ and loss tangent $\left.\tan \delta=0.0027\right)$ with dimension of $0.8 \times 4.1 \times 10.16 \mathrm{~mm}^{3}$. The substrate is placed in the center line of an X-band $(8-12 \mathrm{GHz})$ rectangular waveguide with cross section of $22.86 \times 10.16 \mathrm{~mm}^{2}$. As analyzed in our previous works [26], the couplings between the SRRs and their mirror images generated by the metallic walls perpendicular to the E-field direction can be effectively represented by the equivalent circuits. However, here we mainly focus on how to achieve dual resonance characteristics. The two SRR pairs are separated far from each other and therefore very close to the up and bottom metallic walls (see Figure 1). As a result, the coupling between the two pairs of SRRs can be neglected comparing each pair of SRRs with its mirror image. Moreover, due to the twist angle of SRR pairs, the coupling strengths for the two pairs of SRRs and their up and bottom images are not equal, resulting in the dual resonances properties.

Based on the above analysis and the given restrictions of material and dimensional values, the other parameters of the proposed filter are optimized by finite element method based on HFSS V14.0 software and the results are shown as follows: $d=6 \mathrm{~mm}, r=1.55 \mathrm{~mm}$, and $\theta=40$. Here the parameter $\theta$ can be controlled by some methods, for example, mechanically rotating the single SRR on one of the sides of substrate, which will be discussed later in this paper.

Then, through standard printed circuit board fabrication techniques, the proposed filter is fabricated and placed in the $\mathrm{X}$-band hollow rectangular waveguide as shown in Figure 2. Two waveguide-to-coaxial transitions and the vector network analyzer (Agilent N5230A) are used to measure and record the transmission and reflection characteristics. The thrureflect-line (TRL) calibration procedure is performed to eliminate the system errors during measurements.

\section{Results}

Figures 3(a) and 3(b) show the simulated and measured transmission and reflection properties of the proposed band notch filter. It is seen that both the numerical and experimental transmission curves have two dips, implying a well agreement between the experimental and numerical results. However, there are slight frequency shifts between the measurements and simulations. Specifically, the simulated result shows the transmission dips at $9.725 \mathrm{GHz}$ and $10.025 \mathrm{GHz}$, while the measured result indicates two transmission dips at $9.827 \mathrm{GHz}$ and $10.035 \mathrm{GHz}$. The slight frequency shifts mainly come from the fabrication tolerance in experiments.

Moreover, to further understand the two resonance states of the proposed configuration, Figures 3(c) and 3(d) present the simulated normalized surface current distribution characteristics at such two notch frequencies. It can be seen that the surface currents are mainly concentrated at the upper twist SRRs at lower resonant frequency and contrarily located at the nether twist SRRs at higher resonant frequency. This is because for the lower resonant frequency the larger coupling 


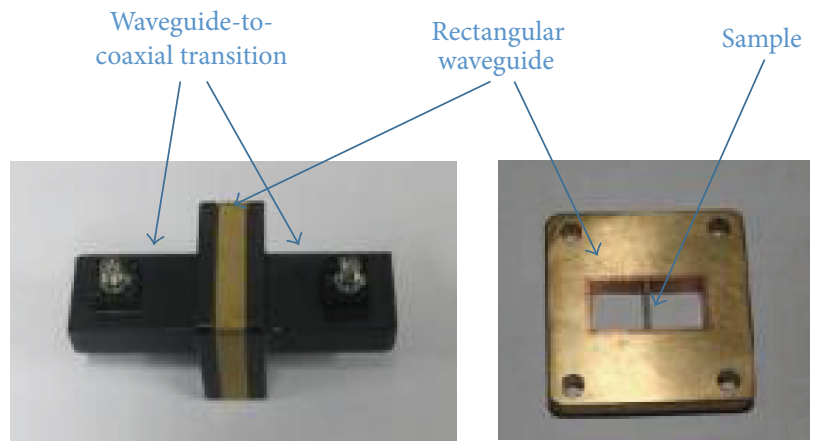

(a)

(b)

FIGURE 2: (a) Measurement setup and (b) fabricated sample inserted in the rectangular waveguide.

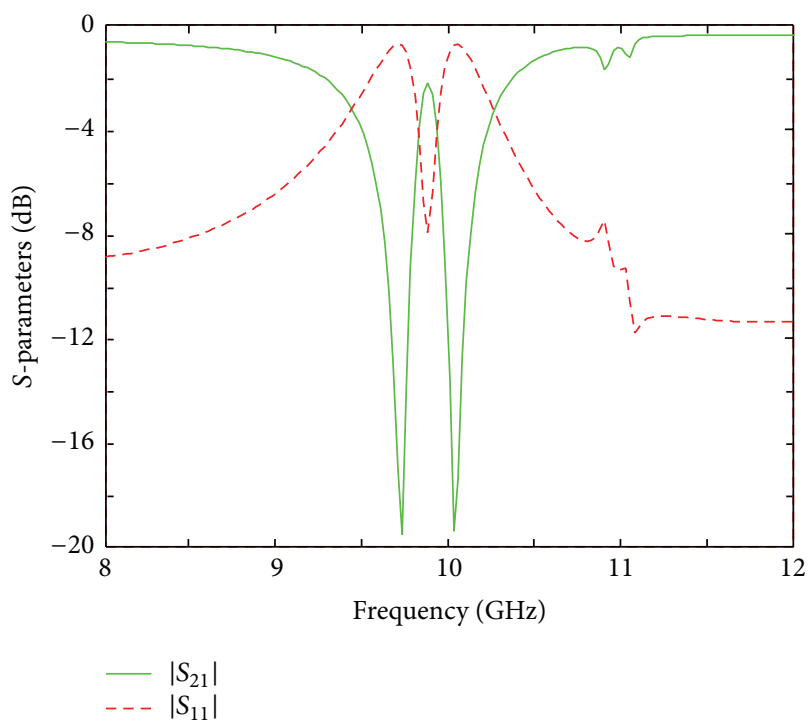

(a)

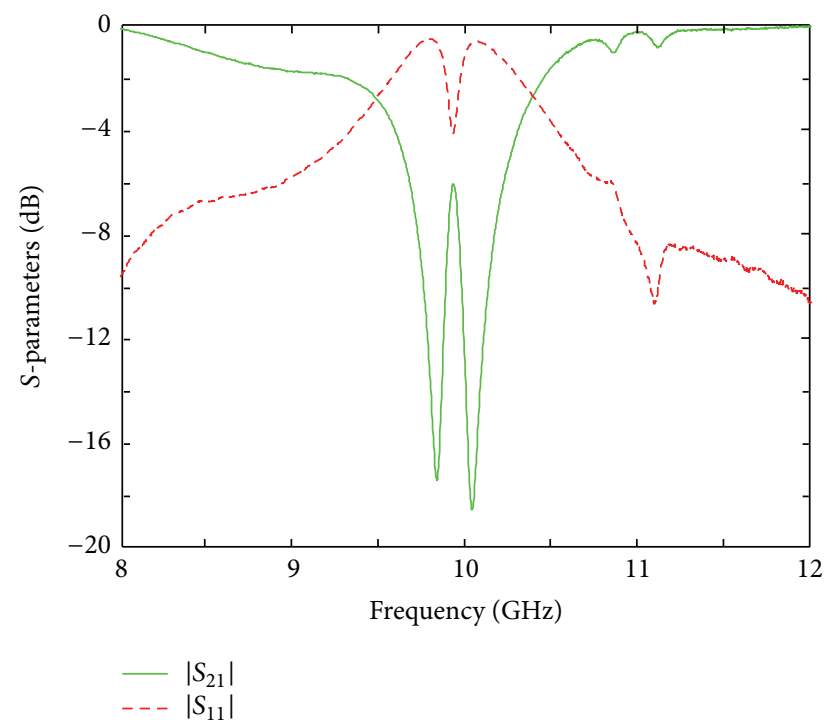

(b)

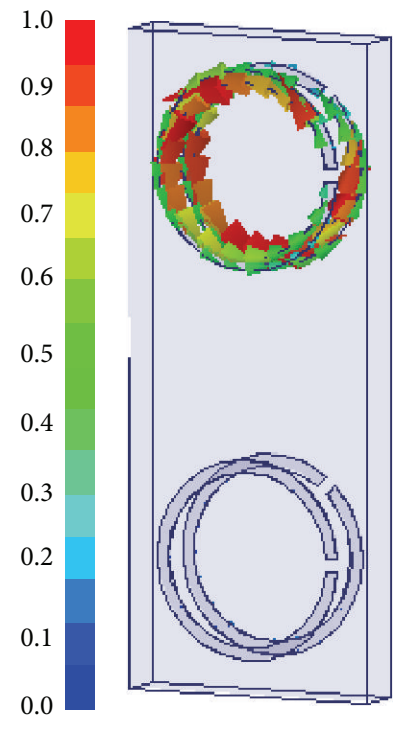

(c)

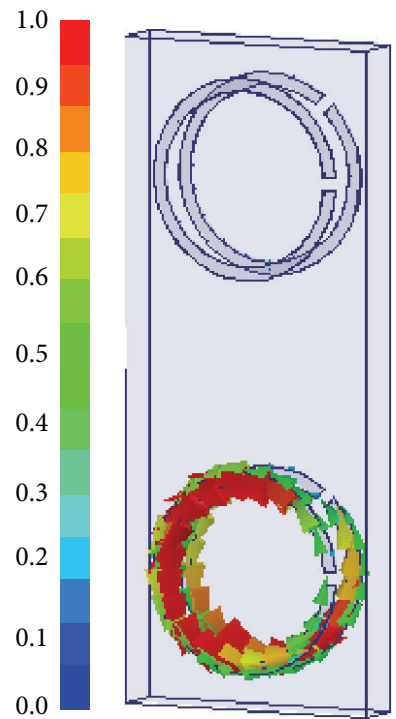

(d)

FIGURE 3: (a) Simulated and (b) measured $S$-parameters of the proposed filter, and the surface current distribution characteristics at notch frequencies: (c) $9.725 \mathrm{GHz}$ and (d) $10.025 \mathrm{GHz}$. 


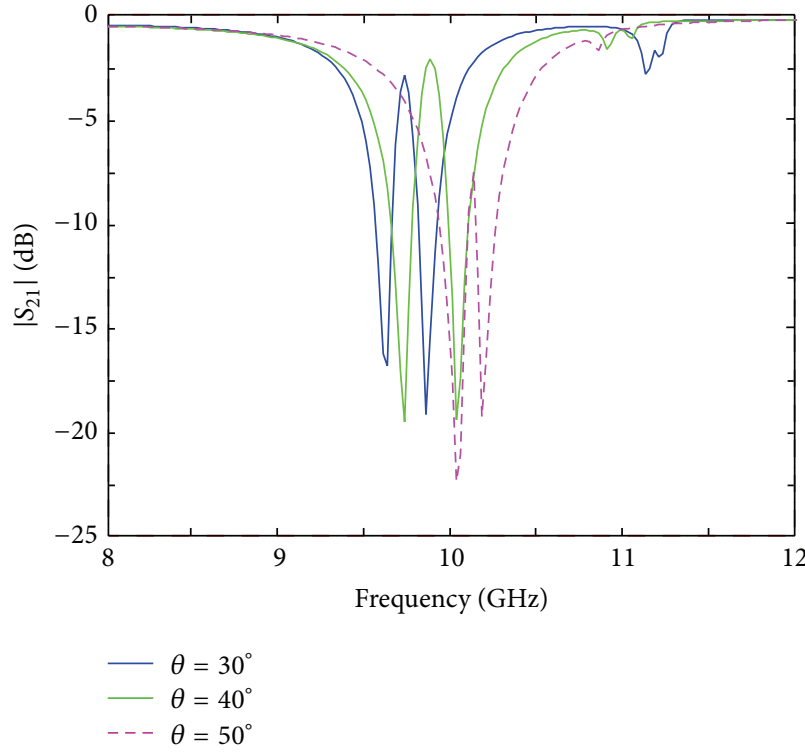

(a)

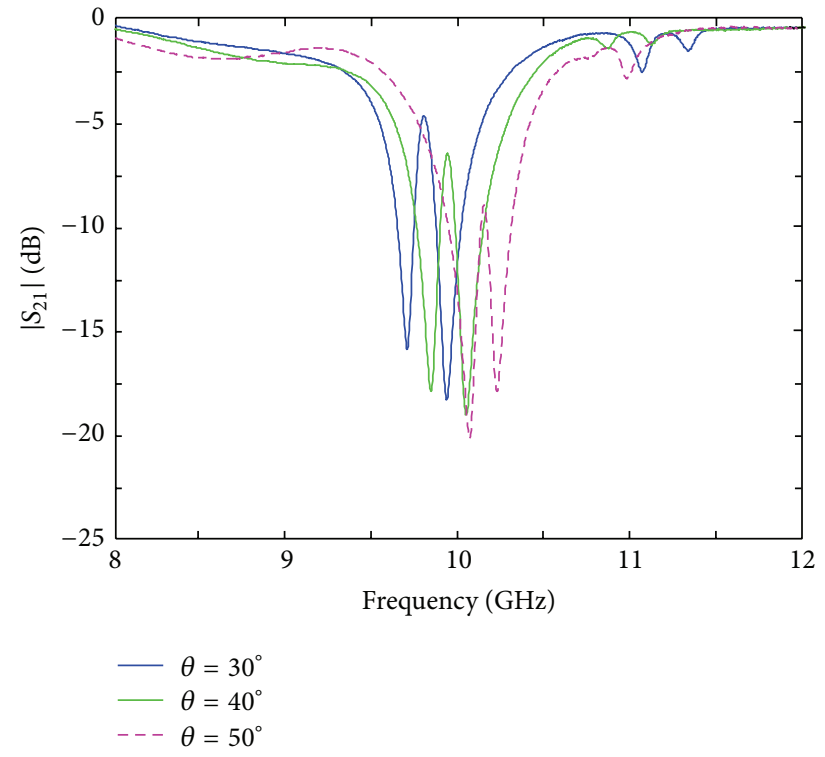

(b)

FIgURE 4: (a) Simulated and (b) measured transmissions of the proposed filter at different twist angles.

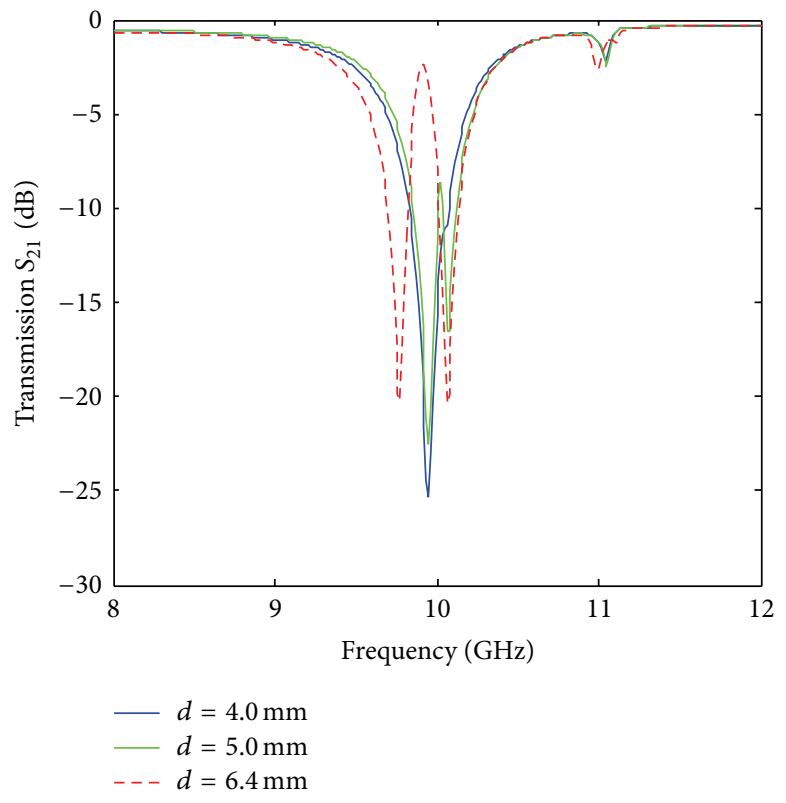

(a)

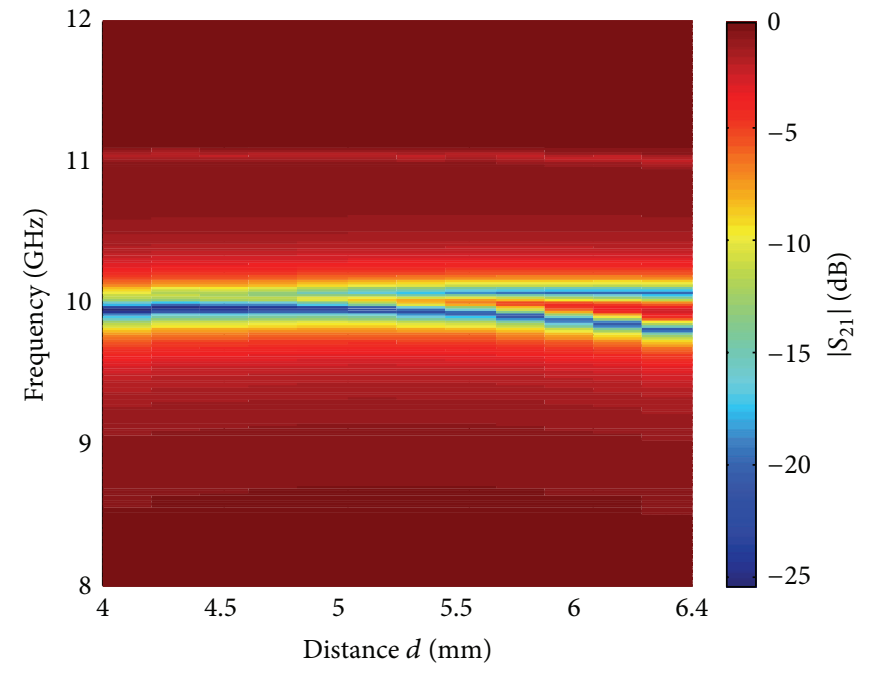

(b)

FIGURE 5: (a) Simulated transmission properties of the band notch filter with three different distance values, and (b) simulated 2D transmission properties with gradually increased distances.

values occur due to the smaller distance between the split of SRRs and the mirror images, compared to the higher resonant frequency. These results agree with the aforementioned analysis very well. Furthermore, as can be observed in Figures 3(a) and 3(b), there are two small transmission dips located in the vicinity of $11 \mathrm{GHz}$. These are the other resonance modes of twist SRRs (come from the inner coupling between the two pairs of twist SRRs) and have little contributions for the proposed filter.
As mentioned above, the operating frequencies of the proposed notch filter can be flexibly controlled by changing the twist angle $\theta$. Here we give schematic measurement results of several fabricated samples with different twist angles. The SRRs on the front side of the substrate are not changed for all the conditions, and the SRRs on the back side are rotated with different twist angles. Also, the upper and bottom twist SRRs maintain the same twist angle $\theta$ for each sample. Figure 4 shows simulated and measured results for the notch filter with 
different twist angles ranged from $30^{\circ}$ to $50^{\circ}$ with step of $10^{\circ}$. It can be known that the simulated and measured results also indicate a well agreement for the notch characteristics. When the twist angle $\theta$ increased, the notch characteristics are not changed and the two notch frequencies are shifted to higher frequency bands. The shifts of notch frequencies mainly come from the changes of coupling strengths between the twist SRR pairs (the front and back SRRs). Specifically, when enlarging the twist angle, the coupling (especially the electric coupling related to the splits within the SRRs) is reduced, resulting in the blue shift of operating frequencies. Also, there are other resonance modes in the discussed twist angles but they do not affect the performance of the filter.

\section{Discussions}

Here we further discuss the effect of distance $d$ between the two pairs of twist SRRs on the couplings and the operating frequencies. As shown in Figure 5, with smaller values down to $4 \mathrm{~mm}$ for the distance $d$, there is only one notching frequency centered at $9.95 \mathrm{GHz}$, and when increasing gradually the distance up to $6.4 \mathrm{~mm}$ two notching frequencies appear with enlarged frequency space between such two operating frequencies. As analyzed similarly in our previous works [26], with closer distance, the differences of the coupling strengths for the two twisted SRR pairs related to the metallic walls of the rectangular waveguide are minimized. Therefore the two operating frequencies are combined to one notching frequency.

\section{Conclusions}

In this paper, a novel dual-band rectangular waveguide notch filter based on the twist SRRs is discussed through numerical simulations and experimental demonstrations. Due to the coupling effects between the twist SRRs and between the original SRRs and their mirror images, the proposed filter exhibits two resonance states and results in the two notch frequencies. Moreover, the two notch frequencies can be controlled by changing the twist angles of SRRs. These results provide a simple way to realize the band notch filter and control the notch frequencies.

\section{Conflict of Interests}

The authors declare that there is no conflict of interests regarding the publication of this paper.

\section{Acknowledgments}

This work was supported by the National Natural Science Foundation of China (Grant no. 61371047) and the Research Fund for the Doctoral Program of Higher Education of China (Grant no. 20110185110014). Yongjun Huang also gratefully acknowledges the Scholarship Award for Excellent Doctoral Student granted by Ministry of Education of China (Grant no. A03003023901006) and the Excellent Doctoral Student
Training Program supported by University of Electronic Science and Technology of China.

\section{References}

[1] N. Engheta and R. W. Ziolkowski, Metamaterials: Physics and Engineering Explorations, Wiley, New York, NY, USA, 2006.

[2] V. G. Veselago, "The electrodynamics of substances with simultaneously negative values of $\varepsilon$ and $\mu$," Soviet Physics Uspekhi, vol. 10, pp. 509-514, 1968.

[3] D. R. Smith, W. J. Padilla, D. C. Vier, S. C. Nemat-Nasser, and S. Schultz, "Composite medium with simultaneously negative permeability and permittivity," Physical Review Letters, vol. 84, no. 18, pp. 4184-4187, 2000.

[4] J. B. Pendry, A. J. Holden, D. J. Robbins, and W. J. Stewart, "Magnetism from conductors and enhanced nonlinear phenomena," IEEE Transactions on Microwave Theory and Techniques, vol. 47, no. 11, pp. 2075-2084, 1999.

[5] J. B. Pendry, A. J. Holden, W. J. Stewart, and I. Youngs, "Extremely low frequency plasmons in metallic mesostructures," Physical Review Letters, vol. 76, no. 25, pp. 4773-4776, 1996.

[6] J. B. Pendry, "Negative refraction makes a perfect lens," Physical Review Letters, vol. 85, no. 18, pp. 3966-3969, 2000.

[7] R. A. Shelby, D. R. Smith, and S. Schultz, "Experimental verification of a negative index of refraction," Science, vol. 292, no. 5514, pp. 77-79, 2001.

[8] S. H. Lee, C. M. Park, Y. M. Seo, and C. K. Kim, "Reversed Doppler effect in double negative metamaterials," Physical Review B, vol. 81, no. 24, Article ID 241102, 2010.

[9] S. Xi, H. Chen, T. Jiang et al., "Experimental verification of reversed cherenkov radiation in Left-Handed metamaterial," Physical Review Letters, vol. 103, no. 19, Article ID 194801, 2009.

[10] B.-I. Popa and S. A. Cummer, "Direct measurement of evanescent wave enhancement inside passive metamaterials," Physical Review E, vol. 73, no. 1, Article ID 016617, 2006.

[11] Y. Feng, J. Zhao, X. Teng, Y. Chen, and T. Jiang, "Subwavelength imaging with compensated anisotropic bilayers realized by transmission-line metamaterials," Physical Review B, vol. 75, no. 15, Article ID 155107, 2007.

[12] W. Zhu, Y. Huang, I. D. Rukhlenko, G. Wen, and M. Premaratne, "Configurable metamaterial absorber with pseudo wideband spectrum," Optics Express, vol. 20, no. 6, pp. 6616-6621, 2012.

[13] O. Paul, Y. Urzhumov, C. Elsen, D. Smith, and M. Rahm, "Construction of invisibility cloaks of arbitrary shape and size using planar layers of metamaterials," Journal of Applied Physics, vol. 111, no. 12, Article ID 123106, 8 pages, 2012.

[14] P. Ding, C. Fan, Y. Cheng, E. Liang, and Q. Xue, "Plasmoninduced transparency by detuned magnetic atoms in trirod metamaterials," Applied Optics, vol. 51, no. 12, pp. 1879-1885, 2012.

[15] H.-X. Xu, G.-M. Wang, and C.-X. Zhang, "Fractal-shaped metamaterials and applications to enhanced-performance devices exhibiting high selectivity," International Journal of Antennas and Propagation, vol. 2012, Article ID 515167, 14 pages, 2012.

[16] H. Liu, S. Lei, X. Shi, and L. Li, "Study of antenna superstrates using metamaterials for directivity enhancement based on fabry-perot resonant cavity," International Journal of Antennas and Propagation, vol. 2013, Article ID 209741, 10 pages, 2013.

[17] T. Tang, "Broad flat-top adjustable filter with metamaterial," IEEE Photonics Technology Letters, vol. 24, no. 4, pp. 288-290, 2012. 
[18] Y. Li, M. F. Iskander, Z. Zhang, and Z. Feng, "A new low cost leaky wave coplanar waveguide continuous transverse stub antenna array using metamaterial-based phase shifters for beam steering," IEEE Transactions on Antennas and Propagation, vol. 61, pp. 3511-3518, 2013.

[19] T. Yang, P.-L. Chi, and T. Itoh, "Compact quarter-wave resonator and its applications to miniaturized diplexer and triplexer," IEEE Transactions on Microwave Theory and Techniques, vol. 59, no. 2, pp. 260-269, 2011.

[20] W. Fei, H. Yu, Y. Shang, and K. S. Yeo, "A 2-D distributed power combing by metamaterial-based zero phase shifter for 60-GHz power amplifier in 65-nm CMOS," IEEE Transactions on Microwave Theory and Techniques, vol. 61, pp. 505-516, 2013.

[21] M. Gupta and J. Saxena, "Microstrip filter designing by SRR metamaterial," Wireless Personal Communications, vol. 71, pp. 3011-3022, 2013.

[22] A. K. Horestani, W. Withayachumnankul, A. Chahadih et al., "Metamaterial-inspired bandpass filters for terahertz surface waves on goubau lines," IEEE Transactions on Terahertz Science and Technology, vol. 3, no. 6, pp. 851-858, 2013.

[23] Y. Zhu, S. Vegesna, V. Kuryatkov, M. Holtz, M. Saed, and A. A. Bernussi, "Terahertz bandpass filters using double-stacked metamaterial layers," Optics Letters, vol. 37, no. 3, pp. 296-298, 2012.

[24] D. Cai, Y. Shang, H. Yu, and J. Ren, " 80 GHz on-ship metamaterial resonator by differential transmission line loaded with split ring resonator," Electronics Letters, vol. 48, pp. 1128-1130, 2012.

[25] Z. Li and Y. J. Ding, "Terahertz broadband-stop filters," IEEE Journal of Selected Topics in Quantum Electronics, vol. 19, Article ID 8500705, 2013.

[26] H. Sun, G. Wen, Y. Huang, J. Li, W. Zhu, and L.-M. Si, “Tunable band notch filters by manipulating couplings of split ring resonators," Applied Optics, vol. 52, pp. 7517-7522, 2013.

[27] J. D. Baena, J. Bonache, F. Martín et al., "Equivalent-circuit models for split-ring resonators and complementary splitring resonators coupled to planar transmission lines," IEEE Transactions on Microwave Theory and Techniques, vol. 53, no. 4, pp. 1451-1460, 2005.

[28] F. Zhang, Q. Zhao, J. Sun, J. Zhou, and D. Lippens, "Coupling effect of split ring resonator and its mirror image," Progress in Electromagnetics Research, vol. 124, pp. 233-247, 2012. 

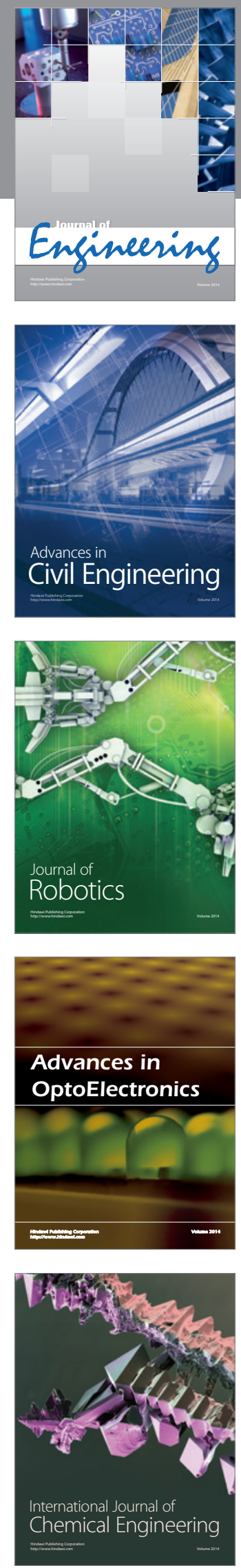

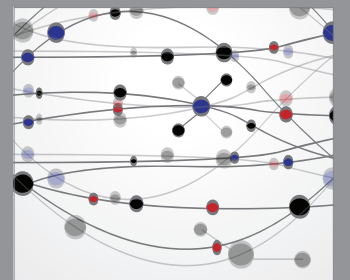

The Scientific World Journal
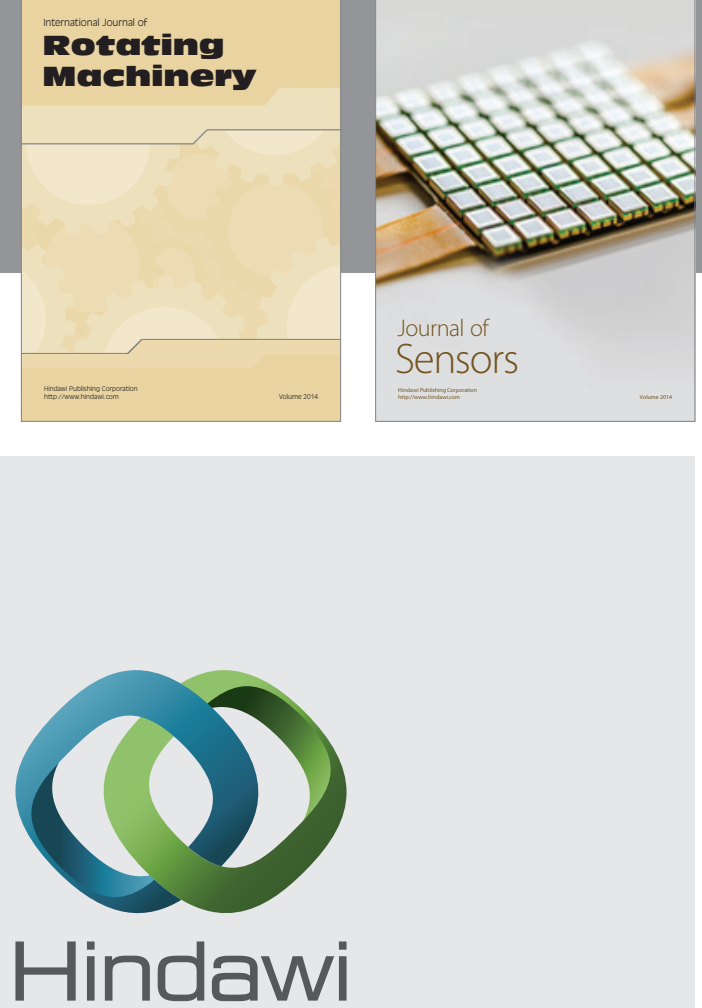

Submit your manuscripts at http://www.hindawi.com
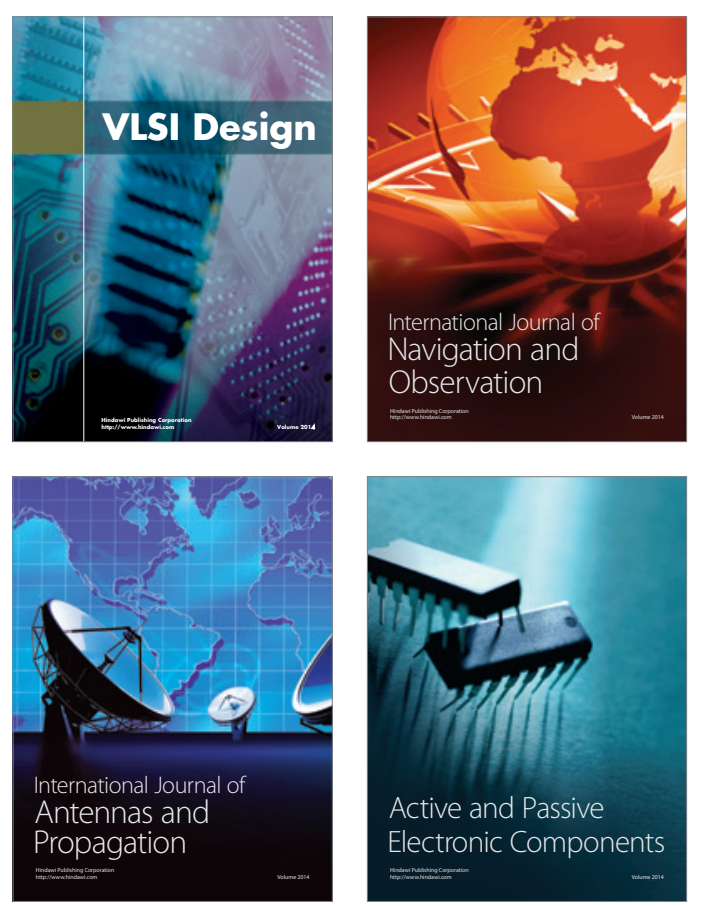
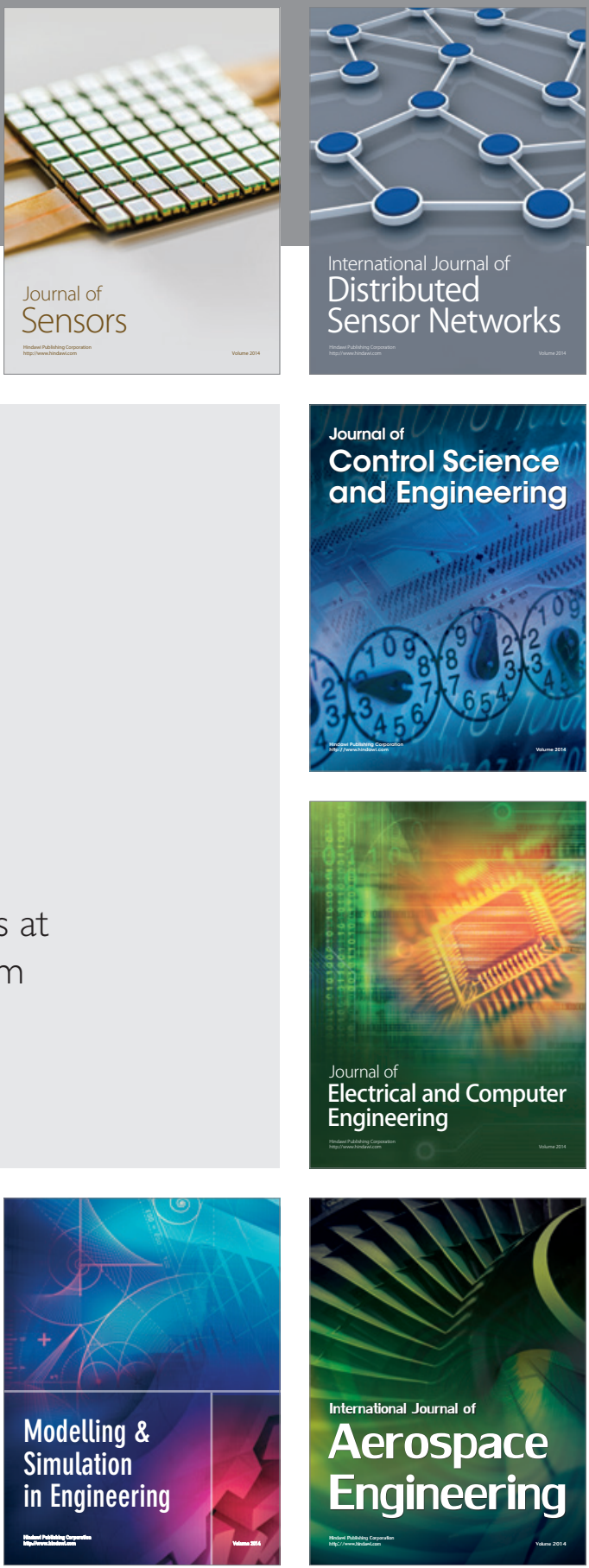

Journal of

Control Science

and Engineering
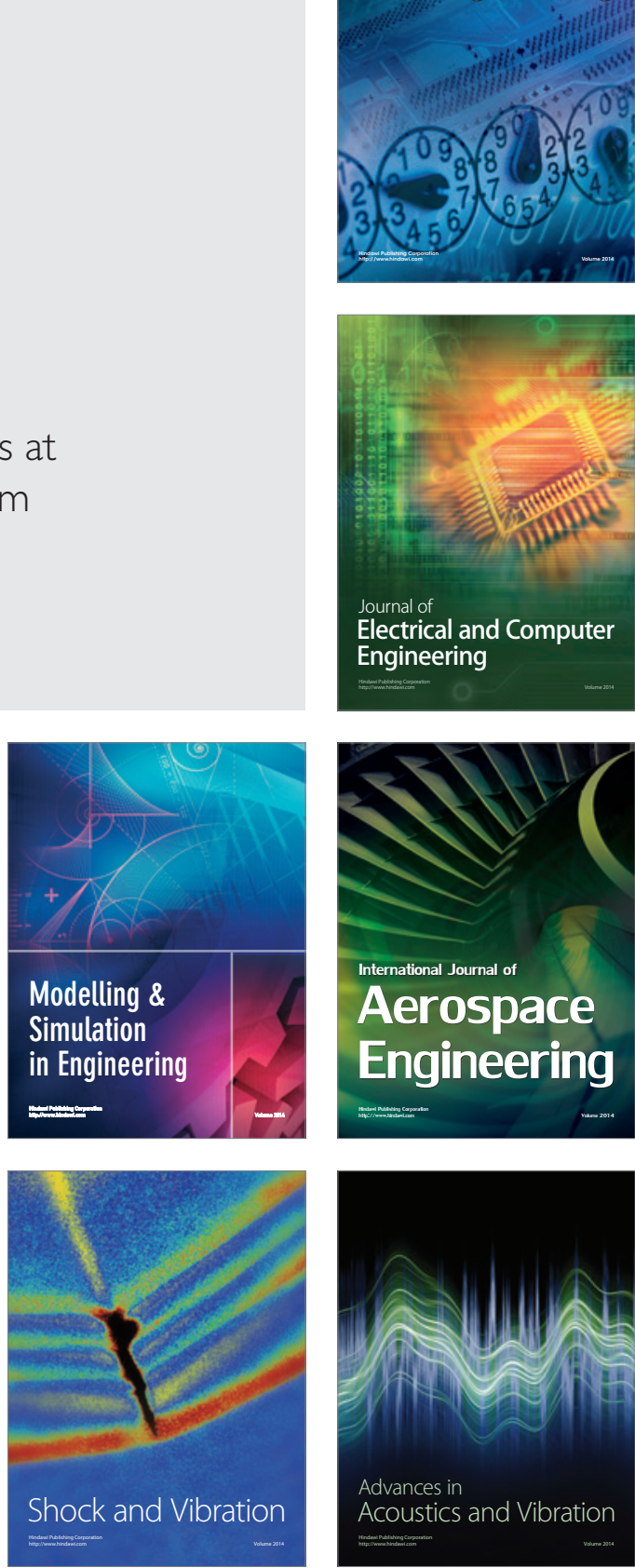